\title{
Design exploration of eBook interfaces for personal digital libraries on tablet devices
}

\author{
Nicholas Vanderschantz \\ Computer Science Department \\ University of Waikato \\ Hamilton, New Zealand \\ vtwoz@waikato.ac.nz
}

\author{
Claire Timpany \\ Computer Science Department \\ University of Waikato \\ Hamilton, New Zealand \\ ctimpany@waikato.ac.nz
}

\author{
Annika Hinze \\ Computer Science Department \\ University of Waikato \\ Hamilton, New Zealand \\ hinze@waikato.ac.nz
}

\begin{abstract}
Mobile eBook readers and reader applications are readily available and are marketed as convenient for accessing personal eBook collections. Different brands of readers and apps are frequently evaluated and compared in consumer, trade, and popular magazines, but rarely in academic studies. This present study examines nine personal digital library (pDL) interfaces, from which design cues are drawn and paper prototypes of $\mathrm{pDL}$ for eBooks are developed. The paper prototypes are evaluated in a usability study to elicit the eBook display preferences of users.
\end{abstract}

\section{Categories and Subject Descriptors}

H3.7. Digital Libraries: User issues;

H5.2. User Interfaces: Graphical user interfaces (GUI)

\section{General Terms}

Design, Human Factors

\section{Keywords}

Personal Digital Library Design, eBook Catalogue Design

\section{INTRODUCTION}

A variety of dedicated mobile eBook readers are available, in addition to reader applications for tablets and phones. Typical interactions with these personal digital libraries (pDL) are closely related to interactions with physical libraries. In both cases, the book borrowing or selection process within a library (digital or physical) may look something like this: the reader searches or browses the collection to identify candidates; the reader examines each candidate book to assess its relevance; and then borrows those books deemed relevant to his/her information need [1]. While this process has been studied little in physical libraries and book shops, it is even less explored for digital environments. This paper investigates peoples' design preferences in personal digital library catalogues (eReaders) on mobile devices. We carried out a survey of nine eBook reader interfaces to assess their design principles. Based on the results of this study, we developed and evaluated paper prototypes for personal eBook library interfaces. Our evaluation identified user preferences in the presentation of bibliographic metadata and visual eBook information, such as book spines, covers and descriptions. The results of our paper prototype evaluation offer insights into people's preferences for

Permission to make digital or hard copies of all or part of this work for personal or classroom use is granted without fee provided that copies are not made or distributed for profit or commercial advantage and that copies bear this notice and the full citation on the first page. Copyrights for components of this work owned by others than ACM mustbe honored. Abstracting with credit is permitted. To copy otherwise, or republish, to post on servers or to redistribute to lists, requires prior specific permission and/or a fee. Request permissions from Permissions@acm.org. CHINZ 2015, September 3-4, 2015, Hamilton, New Zealand

Copyright is held by the owner/author(s).

Publication rights licensed to ACM.

ACM 978-1-4503-3670-3/15/09 ..\$15.00

DOI: http://dx.doi.org/10.1145/2808047.2808054 the design of pDL interfaces on a tablet device.

The remainder of this paper is structured as follows: Section 2 discusses related work on eBook selection and digital library interface design. Section 3 reviews the collection browsing, search features and funtionality offered by the nine mobile book reader applications evaluated. Section 4 explains the methodology of our interface exploration study, and Section 5 presents the study results. The paper concludes with Sections 6 and 7 discussing the results and contributions of our research, along with proposed future work in the area of $\mathrm{pDL}$ interface design research.

\section{RELATED WORK}

The work related to our study falls into two categories: literature on browsing and selection of eBooks (Section 2.1) and on the design of digital library interfaces (Section 2.2).

\section{1 eBook Browsing and Selection}

Researchers have observed the physical actions and eye movements of adults [1], [2] and children [3], [4] when browsing bookshelves within physical libraries. These observed interactions cover a range of physical actions and behaviours. However, little is known about the decision making and cognitive functions of the book selection process in a physical or digital library [5].

Of the limited related work that investigates the book selection practices of adults in a library is [6]. [6] reported that decision making of computer scientists was often guided by book content as well as decisions based on the cover appearance (to gauge book age) and dust (to gauge time since last use). Our own earlier studies of a broader range of adults seeking books in libraries confirmed that decisions are often made based on perceived attributes of the book based on review of covers and spines [2, 7].

From the studies described above we hypothesize that eBook selection similarly might be influenced by book-covers, book-agerelated information, metadata and in-book features such as the table of contents. The study reported here investigates the preferences for presentation of eBook information in a pDL.

\subsection{Digital Library Interface Design}

eBooks are now an accepted medium for recreational reading. In 2011 Amazon's sales of eBooks overtook those of traditional print books [8]. Interfaces for eBook digital libraries on digital devices tend to follow the book shelf metaphor. Digital reading and eBook design research has extensive coverage in the literature e.g. [9][12]. However, most have focused on the design of the book, or understanding the design of books in academic libraries [17] and its contents, rather than the interfaces for cataloguing these books and the browsing of such interfaces.

Similarly research into interfaces for digital libraries, including mobile digital libraries, is sparse and dated. The interface design literature seems predominantly focused on children. For example, the appropriateness in use of the book shelf metaphor in the 
digital environment for children is supported by observations by Moore [4] and Borgmann et al. [13].

Witten et al [14] discuss metadata browsing and the importance of the implicit structure of metadata. Witten et al. describe the use of lists, dates, hierarchies and facets in providing support for different browsing activities. Jones et al. [15] discuss the need for mobile interfaces to be designed with consideration of simplicity, format, context, source and interaction. Because of the small size of the mobile device screen, often interactions and interfaces must be specifically developed for this device format. Jones et al. discuss mobile interfaces as often requiring goal-led navigation and considerate or reduced use of scrolling or paging of content. It must be noted here that the physical size of mobile devices in [16] and [15] was smaller than the design in our own. Jones discusses screen sizes in his work ranging from $2.3 \times 2.3$ inches to $4.3 \times 1.4$ inches. The iPad on which our own study is modelled has a screen size of $7.75 \times 5.82$ inches.

\section{EREADER CATALOGUE INTERFACES}

Here we present an overview of the catalogue interfaces to nine commercial eReader products, to better understand the visual attributes of currently available digital libraries on tablet devices.

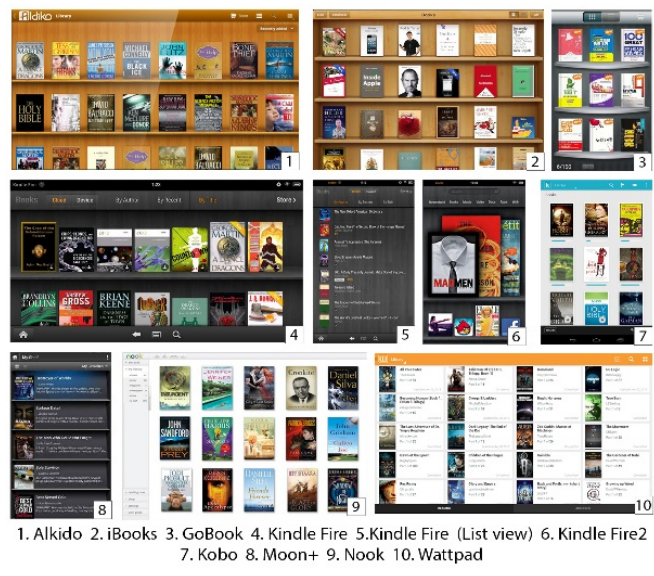

Figure 1: eReader catalogue interfaces.

Aldiko $^{1}$ (only available on Android) and the iBooks interface ${ }^{2}$ (only available on iPad, iPhone and iPod) both use a traditional bookcase theme with brown wooden shelves. At any given time, two to four shelves are in view, depending on screen size and orientation of the device. Books are shown with their cover facing outwards and are scaled to fit within the shelves, with widths and heights proportionate to the printed book equivalent, no indication of the thickness of a book is given. This gives some of an impression of how the book would look in its physical form. An alternative view in Aldiko is also available where each item in the library is shown within a white rectangle that contains the cover, title, author and an indicator of the amount of a book read by the owner. The entries are listed in two columns. The list view in iBooks shows just a cover image and book title.

GoBook ${ }^{3}$ presents books in a stylized metallic bookshelf. The grey/chrome shelves are curved towards the user in a semi-circle shape. Nine book covers are viewable at any one time. All books are represented by their covers and are shown at the same size (height and width), but no indication is given to the thickness of a

\section{http://www.aldiko.com/}

http://www.apple.com/support/ios/ibooks/

${ }^{3}$ https://play.google.com/store/apps/details?id=com.jb.gobook book. A list view is also available, which shows a cover, title, percentage read and date.

The Kindle Fire ${ }^{4}$ displays books using a bookshelf metaphor with book covers in proportion to their physical counterparts. The background of the application is one large, dark grey, wooden shelf. A list view is also available which shows the book covers with titles and author names and percentage read in a list view. Alongside each book cover, its title is displayed in a clear bold font with the author's name in a smaller point size below. The Kindle Fire 2 provides an alternate method for presenting the books on the home display. The book covers are layered on top of one another. A highlighted book is bought to the front of the stack, while the other books are dimmed and stacked below the front book. This interface follows a similar interaction design as the Cover Flow interface made famous by the IOS music library, where users can scroll horizontally through the books. The Kindle Fire 2 also has a list view with the library catalogue, similar to the one used in Kindle Fire, which includes the books' titles and description in clear print alongside the book covers.

Kobo ${ }^{5}$ presents the books in a regular grid of the book covers. This grid might be influenced by the bookshelf metaphor; however, it does not directly reference shelves. Two to four shelves can be viewed at any one time, depending on the device being used and the orientation of the screen (portrait or landscape). The books are all represented by an image of their covers, which are of generic size, and have a shadow around the book image. All books are represented by their covers. Nine to twelve book covers can be seen on the screen at one time. Very thin books, such as books representing newspapers, do not have such a shadow. The alternative view in Kobo still presents a grid of cover images, but each cover is accompanied by its book title and the author to the right of the cover.

Moon ${ }^{6}{ }^{6}$ also uses a grey/silver, metal-look bookcase, and offers two different views of the books. The first view shows up to twelve book covers facing outwards from the shelves. This view cuts off the bottom three books to indicate the ability to scroll vertically. The second view is a list of books, on shelves, with bold clear titles and descriptions located to the right of the covers. Each shelf holds one book and its description. All books are represented by their covers and are a generic size. Moon+ also provides readers with the ability to view the library in a bookshelf methphor layout, similar to that used with Kindle Fire.

Nook $k^{7}$ does not use a shelf metaphor and instead displays the books floating in the air. They are shown in proportion to their physical counterparts and are the covers are presented in a grid in both tablet and smart phone views. Six to fifteen books can be seen at once depending on the device being used.

Wattpad $^{8}$ presents books in two different views. The first view shows the book covers individually with descriptions, reviews, comments and ratings while the second view shows a matrix of several books with the title displayed below each book. The books are displayed in the same size using only a cover.

A core discovery of this survey of eReader interfaces is that the majority of eBook applications use a traditional bookshelf metaphor to present their book catalogue (aldiko, iBooks, GoBook, KindleFire, Moon+). These interfaces were often

\footnotetext{
${ }^{4} \mathrm{http}: / /$ www.amazon.com/Kindle-Fire-Amazon-tablet/dp/B0083Q04IQ ${ }^{5} \mathrm{http}: / /$ store.kobobooks.com/

https://play.google.com/store/apps/details?id=com.flyersoft.moonreader http://www.nook.com

http://www.wattpad.com/
} 
represented by brown, wood-grained shelves. Only a few interfaces used bookshelf-inspired layouts that were visual abstractions rather than illustrative representations of this metaphor. The bookshelf metaphor does however deviate from a physical bookshelf in that all of the covers face outwards, rather than just the stacked spines showing. Some of these applications displayed books at a size proportionate to the physical book, though most kept the book covers inside a generic book shape with a consistent size. The few eBook catalogues that had varying book sizes tended to retain a generic spine depth and width. None of the applications had the spine of the book in proportion to the physical book (KindleFire). Six of the interfaces displayed title information additionally to the information on the cover of the book. Three of these contained further descriptive information. Only a small number of the sampled applications utilized other display methods such as laying books on top of one another (KindleFire2) or "floating" books in whitespace (e.g. nook).

By looking at nine of the catalogue interfaces currently available we identify six key questions:

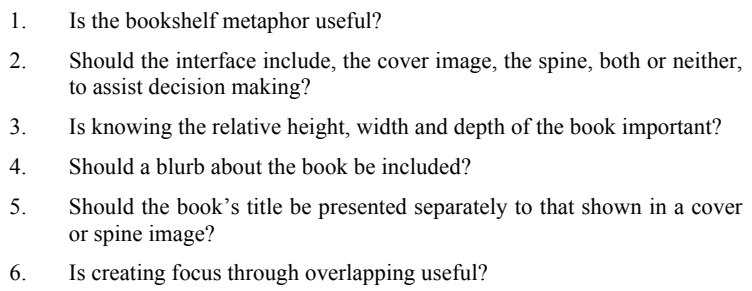

To investigate how readers prefer books to be displayed in a pDL on tablets, we evaluated a range of presentation prototypes. The eBook features explored in the pDL interface prototypes included aspects we identified in the survey, such as the book's spine, front and back cover, and a blurb about the book (meta-data).

\section{TOOLS \& EVALUATION METHOD}

This section reports on the interface prototypes we developed, on the evaluation method and the study sample.

\subsection{Prototypes}

We developed five paper prototype interfaces for eBook catalogues, based on design elements appearing in existing eReaders (Section 3). The prototypes included visual elements such as book spines, front and back cover, and descriptive meta data (title, author) as well as blurbs. These visual elements were created using scans of the covers and spines of books owned by the researcher. All blurbs have the titles in Universe LT Std Bold Condense and body text in Calibri Regular to create readability and consistency across prototypes. The following prototypes were created based on the 6 questions posed at the end of section 3:

Prototype A: Book Cover only (overlapping) (2 \& 6)

Prototype B: Book Spine only (1 \& 2)

Prototype C: Book Title and Blurb only (4)

Prototype D: Book Cover, Title \& Blurb (1, 2 \& 5)

Prototype E: Book Cover Spine \& Back with Blurb (2 \& 4)

We now discuss each of these prototypes in turn. The black \& white images shown are the ones used for the evaluation. Larger image copies are shown in the appendix.

\subsubsection{Prototype Prototype A: Book Cover only}

Prototype A shows the book covers laid on top of one another with the front book highlighted, and all other books darkened. Covers were shown in proportion to the real books, but all books had a generic spine depth. The base of the prototype aims to give a sense of gravity by displaying a dark rectangle under all the books as the ground. Arrows on the right and left edges of the screen indicated the options for horizontal scrolling.

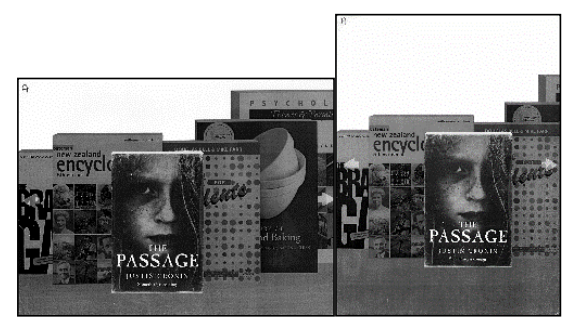

Figure 1: Prototype A in landscape and portrait view.

\subsubsection{Prototype B: Book's Spine only}

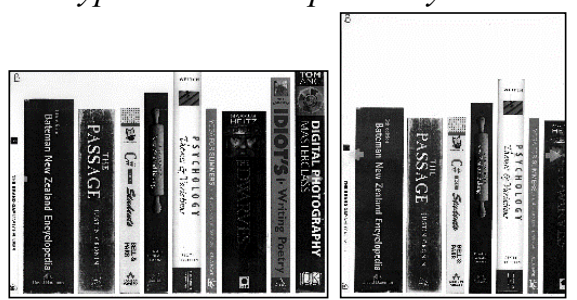

Figure 2: Prototype $B$ in landscape and portrait view.

\subsubsection{Prototype C: Book's Blurb only}

Prototype $\mathrm{C}$ displays just the book blurbs in a generic sized book with a generic sized depth. A grey rectangle on the far left of the application was used to imply a wall to stop users from wanting to horizontally scroll. Arrows indicate horizontal scrolling options.

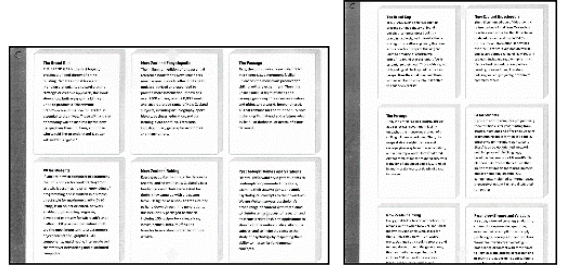

Figure 3: Prototype $\mathbf{C}$ in landscape and portrait view.

\subsubsection{Prototype D: Book's Cover \& Blurb}

Prototype D displays the book front covers with a description and bold titles located to the right of the cover. The book covers are displayed in the same height to width proportions as the real books and have uniform spine depths. A grey vertical rectangle on the left of the screen implies to users that thye cannot scroll horizontally. Arrows indicate vertical scrolling options.

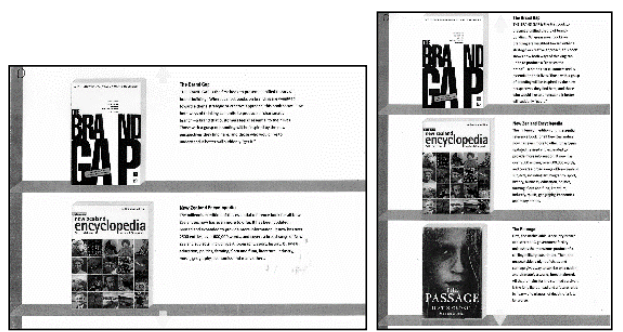

Figure 4: Prototype $D$ in landscape and portrait view.

\subsubsection{Prototype E: Book's Spine, Cover \& Back}

Prototype E displays each book's spine, cover and back in the same proportion as the associated physical books. A grey 
rectangle on the far left of the application is to imply a vertical scroll. The arrows indicate vertical scrolling options.

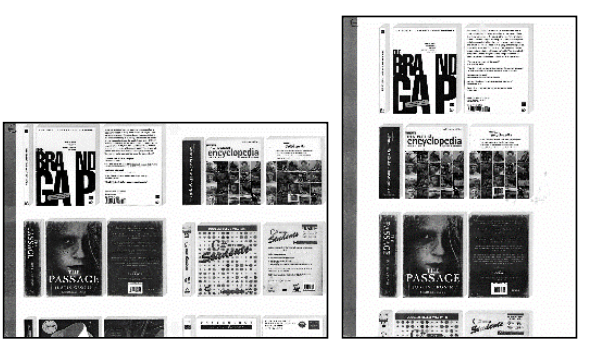

Figure 5: Prototype $\mathrm{E}$ in landscape and portrait view.

\subsection{Study Methodology}

The study was designed to elicit the participants' preferences for the visual display of the books in the pDL and understand how much metadata they prefer when viewing books in a pDL interface. Specifically, the study explored interface design preferences for the display of book spines, front covers, back covers, blurbs and combinations of some and/or all of these elements. The study was performed as a semi-structured interview using the paper prototypes (described in Section 4.1) and six predetermined questions to guide the participants through the interview. The interviews took between 5 minutes and 15 minutes. Only manual notes were taken during the interviews. The six questions asked in the guided interview were:

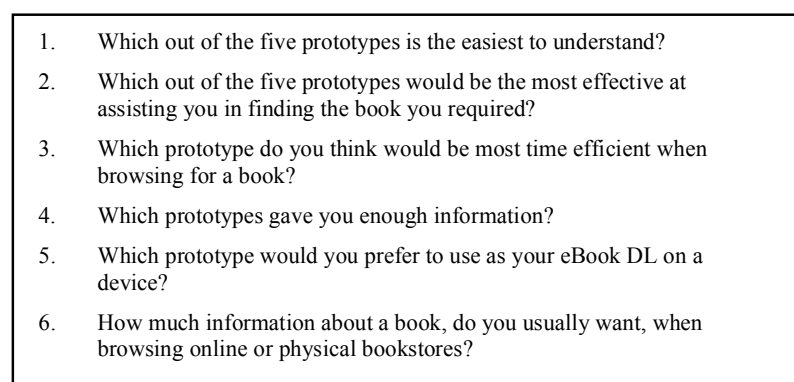

The prototypes were presented to the participants in greyscale (Black and White) as they were paper prototypes rather than functioning interfaces. The participants viewed a portrait and a landscape version of each prototype. A background board was used to support each prototype in the interviews, which had a border image of the Apple iPad 2. This was to create a sense of realism and to allow the interviewee to get an impression of the prototype in its actual proportions. The interviewee took their time to review, analyse and ask questions to the interviewer about the use of each prototype. The prototypes were labelled A, B, C, D, and E. For questions 1, 2, 3 and 5 participants could only choose 1 prototype. For question 4, participants could choose more than one prototype. The questions were intentionally open to interpretation in order to gather broad qualitative feedback. For questions 1 through 5 a "why" probe was used once an interviewee had chosen a prototype. As a probe for question 6, participants were asked to list what information, in their opinion, would fit the criteria.

\subsection{Study Participants}

A total of 60 participants were recruited for this study, 35 male and 25 female. Of the 60 participants interviewed, 50 participants were between the ages of $15-25$ and 10 participants were 25 years and older. Twenty nine participants had completed or were currently enrolled in a humanities or arts degree (i.e., graphic design, humanities, media arts etc), sixteen had completed or were currently enrolled in a science or engineering (i.e., computer science, mathematics, science etc), and fifteen had completed or were currently pursuing multi-major or cross discipline degrees. All particpants were familiar with mobile devices and had some experience reading on a mobile device.

\section{RESULTS}

We present here the results of our paper prototype study.

\subsection{Ease of Understanding}

Question 1 asked which out of the five prototypes is the easiest to understand. Over half of the participants (32) believed Prototype $\mathrm{D}$ was the easiest to understand. Some participants reported that $\mathrm{D}$ was easiest, because the back of the book is not needed to be displayed because the typed blurbs appear far more legible and readable. Participants also felt that display of the spine is not necessary as the title of the book is on the front cover in these prototypes. This simple and clear layout that has both visual and written information about the book (with the title and blurb accompanying the cover) was preferred by participants who liked being able to view the book cover, and then, if they were interested, they could investigate the blurb to discover more details. Participants preferred the visual presentation of the book's physical form to help with recognition. They noted that the placement of the shelves gave them an idea of the collection's layout. Twelve participants commented on how they prefer to see the cover as they believe it provides a visual reference for them

Fifteen participants believed that Prototype A was the easiest prototype to understand. They felt that this layout created a simple focus target for the viewer to keep track of the current selected book. Prototype A was familiar to some participants as they have seen a similar interaction before with the 'cover flow' image display method from Apple's iTunes. This sideways flow felt most familiar to them on a tablet. The large size of the book covers made participants want to click on the books with the expectation of finding more information about the book.

The seven participants who believed prototype B was the easiest to understand felt the view of the book spines reminded them of a library. The participants appreciated being able to visually "see" the physical form of the book, and noted that the books were large enough to clearly read the titles. The layout encouraged participants to want to click on a book spine and be presented with more information. One participants noted that this prototype allowed them to see the most books from the collection on the screen at any one time.

Six participants chose prototype E, as they felt that the covers were clear enough to read and that with a single glance they could see all possible information they would require. Participants felt that the prototype was more readable when viewed from the portrait perspective. One participant commented that this perspective presents a better visual unity and, additionally, seeing the spine, cover and back gives them the full information of the book's physical appearance. They liked that the prototype offered a lot of visual and written information about a book on one screen.

\subsection{Finding Books}

Question 2 asked which out of the five prototypes would be the most effective at assisting you in finding a book you required. Prototype D was considered by 27 people to be the most effective prototype at assisting the participant in finding a book. Participant P1 explained that they found this prototype to be simple and quick to scroll through as the cover and description gave them visual and written information about the books. One participant (P6) 
observed the books to be clearly separated with the covers and blurbs close together unifying them as a part of the same book. Two participants (P47, P57) found the clear blurb better for searching than the back of a visually-crowed book. With one of these participants stating that they found the blurbs more readable than in prototype E. Further questions revealed that these participants are more likely to associate books with their covers than titles, making this prototype the preferred choice. Five participants observed that this prototype has multiple visual identifiers that all assisted with searching. One participant (P18) commented that using both the visual and textual aspects of the book is helpful.

The 16 participants who believed Prototype B to be the most effective to assist in finding a book noted among other things that it was the clear and straight forward order as well as the advantage of having the most books on the screen at once that made this design appealing. Participants P5 and P9 liked the similarities between this prototype and library shelves. Two participants (P22 and P50) commented that the view of the spines allowed them to effectively search through the books by only seeing their titles and not have copious amounts of information about the books. Participant P59 said they enjoyed the interaction of flicking through the book spines.

Seven participants believed that Prototype A was most effective in assisting with finding a book. Participants P12 and P56 assumed that if the collection was a personal eBook DL, then they would have a rough idea of what a book looked like. This would speed up the process of finding a book as there are many covers displayed at one time on the device and it might be easier to look for a title and/or pictures on a cover rather than a description. Participants P21 and P31 preferred this layout of the books as it made scrolling through the covers easy.

Eight participants believed that prototype E was the most effective prototype for assisting finding a book. Comments from participants included that they enjoyed the large amounts of visual imagery (P11) and been able to view multiple books at the same time (P13). P13 explained that due to the number of books displayed at once, the amount of scrolling required to get through the collection would be less than in other prototypes. The books displayed were found to be easy to differentiate and read. Participant P36 found they were able to gather the title and information required from the images of the books. They described how the spine view and cover helped them in their recognition of books. Two participants believed that Prototype $\mathrm{C}$ was the most effective prototype for finding a book. Participant P45 believed the blurb of the book had more information than a cover. P58 found it faster to search with all data in the prototype being descriptions, as they felt that scanning through text would be more efficient than searching through book covers. P45 also observed that this prototype is able to display a larger number of books on screen compared to prototypes D and E.

\subsection{Time-Efficient Browsing}

Question 3 asked which prototype do you think would be most time efficient when browsing for a book? 22 participants believed that prototype $\mathrm{B}$ was the most time-efficient prototype when browsing for a book. Participants made a range of comments about the benefits of prototype B, including that it had clear visual information (P3), which supported easy browsing. Nine participants felt that this prototype supported time efficient browsing because of the number of books they could fit on one screen (compared to other prototypes). P7 liked that the representation of the book already gives an idea of how long the reading of the book might take and a clear view of where the book starts and ends. The books are only represented by images of their spines; this allows the participant to quickly scroll through the DL. Four participants (P3, P23, P36 and P45) all observed that the spine of a book can fit a lot of information including the title and image reference of the book. Participant P26 commented on the association of the prototype and the bookshelf metaphor. Participants P38 and P43 described how the spines layout allowed them to easily navigate through the collection. Participant P34 noted that if the pDL contained a personal book collection then they would be able to quickly zoom through the collection to find a book as they would have some idea of what spine they would be looking for, making this prototype optimal for speed.

16 of 60 participants believed that Prototype D was the most timeefficient prototype. Participants P5 and P39 liked that this prototype presents the information from the back of the book in a clear and legible format. Participant P36 observed the prototype to have a minimal, yet sufficient, amount of information compared to the other prototypes. Prototype D was considered by P31 to allow them to quickly scroll through covers and/or descriptions. The brief description about the book accompanying the cover was appreciated by P13 as they thought it would help to make informed decisions on whether or not the book is appropriate for their need. The design feature of Prototype D, including the clear grid and whitespace surrounding the titles, was mentioned positively by participant P22 as it allowed them to easily focus on the titles.

Five participants believed that Prototype $\mathrm{C}$ was the most time efficient prototype when browsing. Participant P2 felt this prototype offered all the information they would need, "all information is there and in front of you". The descriptions in this prototype were preferred by P28 and P47 especially when the cover is unknown, preferring to investigate what the book would be about in the description rather than to judge a book by its cover. Participant P32 observed that the blurbs did not take up much space on screen, making it possible to view 6 books at one time. This participant (P32) felt that this allowed them to quickly scroll through the collection.

Fourteen participants believed that Prototype A was the most time efficient prototype when browsing for a book. Participant P1 felt Prototype A gave a sense of being in a library or a shop because it displayed a lot of covers on a shelf. P1 also felt that being able to swipe through the covers, they could see a lot of possible books at once. If the user has an idea of the book cover in mind, participant P53 felt this method would be efficient to locate this book, because this prototype has the ability to highlight a book within the catalogue making the books faster and easier to locate. P53 also stated that due to the simplicity of the prototype (using only the book covers as identifiers), they were able to take in the visual information faster than the written information about a book. Participant P18 felt covers were fast to flick through especially on the landscape view as there are more covers visible. Participant P35 commented that by simplifying the representation of a book to its cover, this gives enough indication what the book would be about, however if it was simplified more (e.g., only viewing the spine), then the title and thickness would not be sufficient to indicate the content.

Three participants believed that prototype E was the most timeefficient prototype when browsing for a book. Participant P54 selected $\mathrm{E}$ because they preferred to have a large amount of both visual and written information about one book presented at one time, while being able to view 4 books on screen. They also 
found the information to provide enough detail without having to read a whole paragraph. The imagery was deemed sufficient by P56 to identify the topic leaving enough space for the user to scroll through several books without becoming overburdened by lots of content.

\subsection{Sufficiency of Information}

Question 4 asked which prototypes gave enough information. Participants were allowed to select more than one prototype if they felt that more than one fitted the criteria. A total of 109 votes were collected. 53 of 60 participants believed that Prototype D gave enough information about the books in the DL. Five participants stated that Prototype D contained a good balance of visual and written information about the books. If the book in the DL is known then P1 felt that visual recognition of the cover is all that would be needed. The description in prototype $\mathrm{D}$ was said to be sufficient to give an in-depth view of the book without having to investigate the content of the book by 21 participants. The description introduces the viewer to its content while explaining the cover the users are looking at. According to comments made by participants, Prototype D fulfils both the visual requirements of those who prefer to judge a book by its cover and those that prefer to see details about the book before choosing it. Prototype D offers two ways of viewing the title of the book, one seen on the cover of the book and the second seen above the description. Participant P5 felt that prototype D was best for looking at titles. One participant noticed that Prototype D offered the same information as Prototype $\mathrm{C}$ in a clearer layout, and that Prototype $\mathrm{D}$ was not as cluttered as prototype $\mathrm{E}$.

27 participants believed prototype E gave enough information about the books in the DL because the back of the book had descriptions (P42) and the title was repeated within the images to help the user visually recognise the books (P40). Participant P44 liked how the images gave an impression of the appearance of the physical books. 24 participants believed Prototype C gave enough information about the books in the DL. Seven of the participants commented that Prototype C contained enough written information about the books. Participant P55 particularly mentioned that blurb could stand on its own without the need to be accompanied by an image. Participant P3 felt the clear title and readable blurb was more useful than an image of a back of a book. They also felt the blurb to be more informative than a cover image. 3 participants (P20, P30, P48) believed Prototype A gave enough information about the books. The participants stated that for them the cover gave enough visual and textual information, especially when it included the title and author.

2 participants (P30 \& P42) believed prototype B gave enough information about the books in the DL. They observed that the spine offered the title of the book, which was the textual information they required, along with the visual reference.

\subsection{Preference on a device}

Question 5 asked which prototype participants would prefer to use for a $\mathrm{pDL}$ on a mobile device. 31 participants preferred Prototype $\mathrm{D}$ to be used in their pDL on mobile a device. Eight participants who preferred D stated that the presentation of the covers was an influencing factor in their preference. Participant P2 commented that readers used to seeing book covers as visual representation and descriptions alongside the image. Participants P1, P3 and P26 felt that the whitespace surrounding the image and text made the layout easy to navigate. Participants P9, P31, and P42 preferred the landscape view as they felt the design to be enhancing the books in this view and closely resembled a bookshelf. Nine participants found the cover to be an easy visual identifier and commented that this was useful in combination witht the the written information, in a simple format (P51). Participant P57 felt the unidirectional scroll and appropriate mix of enough information allowed them to familiarise themselves with the book's visuals while being reminded of the book's content through the description.

14 participants believed that Prototype A was the interface they would want for their pDL on a device. Participants P12, P40 and P44 found the visual orientation appealing, particularly as they liked seeing the front covers. Participant P20 thought that this prototype looked "prettiest" and felt they got a better impression of the book when they saw the cover, preferring pictures over descriptions. Participant P60 felt the size of the covers made it easy to read the titles of the books. The scrolling feature was felt by P27 to make the books more accessible. For P56 the colours of the books acted as visual cues, "All laid out, the colours are visual cues indicating the books there, when scrolling through, selected books would come to focus like a gallery". Participant P36 assumed that if they knew the collection, they would only need the covers to find a preferred book as they would visually recognise it. Most participants that chose this prototype commented that they were visual people.

10 participants believed that prototype B was the prototype the participant would want as their pDL on a device. Participant P8 selected this prototype as they preferred the tidy layout, making the selection of a book easy. The landscape view was reported to be more effective as it resembled a natural bookcase or a library P9, sayin it makes them "feel felt at home". Being able to see multiple books on screen was said by P42 and 56 to make viewing the collection a colourful experience, acting as visual cues.

Five participants believed that prototype $\mathrm{E}$ was the one they would want for their $\mathrm{pDL}$ on a device. Participant P25 chose this prototype because they preferred being able to see four books at once; they felt this allowed them to look for a book quicker than on some other prototypes. Participants liked the range of visual information offered: P28 noticed that the cover drew them into the book and additionally that they could find out more about the content of the book from the description on the back cover.

\subsection{Information Density}

Question 6 asked how much information about a book participants typically want when browsing online or physical bookstores. A total of 32 items of information were collected from the 60 participants. We report here the 10 most popular items, all of which were mentioned by at least 5 participants. The book's blurb was the most popular piece of information the participants wanted when browsing online or physical bookstore; it was mentioned by 50 out of the 60 participants. 37 participants requested the book covers, 22 the book titles, 21 the book authors, 12 participants mentioned the back of the book, 12 participants mentioned the book's genre, 11 participants wanted to see reviews, 8 participants wanted to see the price, 7 participants want the year the book was published and 6 participants want the publisher's name. A total of 22 other suggestions were made by participants, these included; ratings, example pages, recommended books, number of pages, edition, cost, popularity and whether it was a best seller.

\subsection{Improvements suggested by participants}

Participants suggested modifications or options for Prototypes A and B that would allow for showing a blurb upon user interaction. It was suggested that a book might flip to show the blurb in the space currently allocated to the book within the interface for Prototype A rather than moving to an intermediary page, for 
Prototype B it was suggested that the books would separate to allow for this information. Alternatively it was also suggested that Prototype A \& B could benefit from an intermediary screen similar to the interface of Prototype D upon user intervention. This intermediary screen would display an image of the book selected and a description next to it.

Modifying the display of the spines of books to horizontal spines stacked on top of each another for the portrait view of Prototype B. Participants noted that they would like to be able to read the spines more easily when they flip the screen.

It was suggested that Prototype D in landscape could benefit from horizontal scrolling as compared to vertical scrolling. Combining the cover images of Prototype E and the blurbs of Prototype D to create a prototype that displays the physical cover as well as the blurb was proposed. A 360 degree representation of the physical form of the book was proposed for Prototype D.

It was suggested that the incorporation of a zoom function on Prototype E would enable easier use of the back of the book. A development of an indication of how many books are currently in the DL was also suggested.

\subsection{Further Observations}

There was no clear distinction between prototypes chosen by males and females, nor was there a distinction dependent upon age, qualification or subject of study. The majority of participants declared during the interview that they do mostly judge a book by its cover and would prefer to see the cover rather than solely a description of a book. Most people preferred the landscape view of the tablet. About a quarter of those interviewed used both the landscape view and the portrait view of the prototypes. Only one participant went through the guided interview using the portrait view only.

Prototype A held strong association for people who had previously used Apple products. Most participants recognized the similarities of Prototype B to a bookshelf at home or the library. People chose this prototype for its ability to display so many books at once on one screen. Prototype B was chosen as the most time efficient prototype. A wide range of participants liked the idea of scrolling through the spines however they would want interactions to discover more about the book as well as more visual information.

When asked about sufficient information, many participants declared Prototype $\mathrm{C}$ had enough or in some cases too much information compared to Prototype D. However the blurbs presented on Prototype $\mathrm{C}$ were the exact same amount of text as were presented on Prototype D. Only one participant of the 60 noticed that the text was the same. We believe participants may have interpreted the use of the term information in this question as equating to written data. It is plausible that participants may have assumed that Prototype $\mathrm{C}$ contained different content to Prototype $\mathrm{D}$ and the presentation differences in these two prototypes may be indicative of the type of presentation preferences for blurbs.

Prototype D was the only prototype with copious amounts of whitespace. This was because the design of the prototype would not allow 3 or 4 books to comfortably sit on the landscape view. It came as a surprise that people tended to favour Prototype D because of the whitespace surrounding the books. Even though the prototype lacked the capability to show more than two books at a time, people enjoyed the comfortable space surrounding the information and chose the prototype as their personal favourite. The visual aesthetics within Prototype D used simple thin rectangles to separate the book's information from one another, which helped people associate the blurbs to the books they belong to. This association created a strong presence of the book, allowing it to be fully identified with the visual and written information present on screen at the same time.

Prototype E offered participants the ability to see the entire book on screen. Prototype E did offer more books on screen then Prototype $\mathrm{D}$ but participants had trouble reading the details of the books and would discard this prototype in favour of Prototype D because of the readability and legibility of the data. It would seem from the guided interviews that a combination of Prototype B and Prototype D would provide the most favourable interface for an eBook DL.

\section{DISCUSSION}

We summarise our findings, discuss some observations and comparisons with other studies and limitations of the evaluation.

\subsection{Summary}

From the feedback of the 60 participants we conclude that Prototype D was the preferred prototype in five categories: easy to understand, effective browsing, time-efficient, with sufficient information and overall preferred on device. It was found to be easiest to understand as it offered a clear layout providing both visual and textual information. People preferred a separate short description (blurb) over seeing the back of the book, and similarly seeing both the book and the title re-printed for clarity. Prototype $\mathrm{D}$ was also found to be the most effective prototype in assisting people to find a book they require, using both visual and textual information (cover, re-printed title and blurb). Participants felt that these aspects worked particularly well in portraying the book's content, look and feel. It presented sufficient information as the cover image with visual memory with further advantage of having the title and the description clearly repeated. Participants wished to use Prototype D on a device. The only category in which a prototype other than $\mathrm{B}$ was preferred, was for timeefficient browsing. Participants remarked that the spine of a book can offer a lot of information including the title and image reference of the book, and B allowed many spines to be shown to quickly scroll through the DL. Participants found Prototype B more of a time saver compared to D, through its ability to display enough information about a book and have a large quantity of books seen at one time on the screen. The most important information for the participants when browsing online or physical bookstores, was the book's description. Other information items mentioned were cover, title, author, a view of the back of the book, the book's genre, some reviews on the book and the price.

For Question 1, participants took more time to examine all of the prototypes, compared to any other question. This might be because participants were first familiarising themselves with all of the prototypes. We do not believe the order that a participant was handed the prototypes was a factor in this research. Some participants appeared to find a prototype that they preferred initially and retain this as their prototype of choice independent of the questions that followed. It appeared that participants identified a prototype that they preferred overall, and this prototype was often reported as the prefered prototype for each of the 5 preference questions that followed. For Question 2, some participants wanted to know whether the book collection was their own or if it was a bookstore collection. Participants wanted less information and more visual representation for their own collection but a more informative prototype for an online DL. Participants commented on wanting a search bar if the collection was very large such as a bookstore. Many participants stated a preference for a system similar to Google, instead of having to 
scroll through a list of objects, when the collection is very large. Question 3 was the only question where participants tended to select a unique prototype compared to that discussed in other questions. It was the only question where Prototype D was not the overall most favoured. People believed viewing the book's spines would be optimum for an efficient search as many people are familiar with this system in libraries and in their own homes. For

\subsection{Comparison with other studies}

As per Witten et al.'s advice [14], our study proves that the addition of metadata such as title and description alongside the visual representation of the book (its' cover) is preferred by the pDL users in this study. The users in this study were primarily students, but were not necessarily considering the interface for academic purposes. The need for a visual cover presentation may differ in an academic, rather than leisure context.

Borgman et al. [13] showed for children that the bookshelf metaphor was a successful tool for facilitating effective browsing of digital library catalogues. However, our own study did not target children and showed that for adults interviewed, the rigid or visually realistic use of a bookshelf metaphor is not in keeping with users' preferences. This is contrary to common industry practice, as shown in our survey (Section 3), where many current eBook systems utilise a visual bookshelf metaphor. The general preference for prototype D may be able to be attributed to it's combination of a cover and blurb, giving a range of metadata as well as a layout that combines plenty of white space and results in an uncluttered interface that does hint at the bookshelf metaphor.

The result that the spine only interface was chosen most often when efficacy of information presentation was considered aligns with Jones et al. [15] who highlight the need for limited scrolling on mobile devices. The reason for this may be that our prototype was percieved by users to have the familiarity of a bookshelf metaphor, while different to the metaphore usually implemented and also giving a sense of being 'time efficient' for the user. Conversely, it is unsurprising that the interface with only two visible books, but additional metadata (prototype D), was chosen for all other questions asked.

A recognised limitation of this investigation is that presently we have tested paper prototypes. This of course controls for potential variables of digital interaction which may influence the results. Our next step is to develop digital and functioning prototypes based on our findings to elicit further understanding of display preference for $\mathrm{pDL}$ during an interactive scenario study.

\section{CONCLUSION}

This paper explored interface and layout options for a personal digital library and eReader software. We executed a survey of existing eReaders and compared nine software interfaces. From the resulting feature list, we created five paper prototypes for interfaces, which were explored in a user study with 60 participants. We conclude that the stale metaphor driven interface design trend noted in pDL design on mobile devices does not meet the needs or preferences of eBook users today.

The interviews found that most people preferred an interface that encompassed book covers, bold titles and a book description. This interface was identified by more participants when asked if it was easy to understand, effective for finding books, and had sufficient information. Further, participants considered the design to be clear and informative. For fast search, an interface with only the spines of books displayed was preferred. It is also apparent that the interface required for a pDL of eBooks that the user owns is different to the interface required for books for purchase.
We will further this work with future studies that investigate the dynamic aspects of mobile pDL interfaces during task specific interactions. Interactive prototypes based on this work will alow us to assess the impact of functional buttons, scrolling and navigation on users preferences when interacting with eBook collections in pDLs.

\section{Acknowledgements}

We acknowledge and thank our researcher Dairne O'Sullivan who worked with us on the prototype design and conducted the interviews and initial analysis for this work.

\section{REFERENCES}

[1] D. McKay, A. Hinze, R. Heese, N. Vanderschantz, C. Timpany, and S. J. Cunningham. 2012. An exploration of ebook selection behavior in academic library collections. TPDL 2012, 13-24.

[2] A. Hinze, D. McKay, N. Vanderschantz, C. Timpany, and S. J. Cunningham. 2012. Book selection behavior in the physical library: Implications for ebook collections. JCDL 2012, 305-314.

[3] D. R. Reutzel and K. Gali. 1998. The art of children's book selection: A labyrinth unexplored. Read. Psychol. Int. Q., vol. 19, no. 1, 3-50.

[4] P. Moore. 1995. Information problem solving: A wider view of library skills. Contemp. Educ. Psychol., 20 (1), 1-31.

[5] I. Rowlands, D. Nicholas, H. R. Jamali, and P. Huntington. 2007. What do faculty and students really think about e-books? Aslib Proceedings, 2007, Vol. 59 No. 6, 489-511.

[6] H. Stelmaszewska and A. Blandford. 2004. From physical to digital: a case study of computer scientists' behaviour in physical libraries. IJDL4(2), 82-92.

[7] Hinze, A., Alqurashi, H., Vanderschantz, N., Timpany, C., \& Alzahrani, S. 2014. Social Information Behaviour in Physical Libraries: Implications for the design of digital libraries. JCDL 2014, 107-116.

[8] M. Hamblen. 2011. Amazon: E-books now outsell print books Computerworld, 19-May-2011.

[9] M. Landoni and F. Gibb. 2000. The role of visual rhetoric in the design and production of electronic books: the visual book. Electron. Libr., 18(3), 190-201.

[10] J. Nielsen. 1990. The art of navigating through hypertext. Commun. ACM, vol. 33, no. 3, 296-310.

[11] F. Crestani and M. Melucci. 1998. A case study of automatic authoring: from a textbook to a hyper-textbook. DKE 27(1), 1-30.

[12] R. Wilson and M. Landoni. 2003. Evaluating the usability of portable electronic books. ACM Symp.Applied computing, 564568.

[13] C. L. Borgman, S. G. Hirsh, V. A. Walter, \& A. L. Gallagher, 1995. Children's searching behavior on browsing and keyword online catalogs: the Science Library Catalog project. JASIS, 46(9), 663 684.

[14] I. H. Witten, D. Bainbridge, and D. M. Nichols. 2009. How to build a digital library. Morgan Kaufmann.

[15] M. Jones and G. Marsden. 2006. Mobile interaction design. John Wiley \& Sons, 2006.

[16] S. Jones, M. Jones, and S. Deo_andA. 2004. Using keyphrases as search result surrogates on small screen devices. Pers. Ubiquitous Comput., vol. 8, no. 1, 55-68.

[17] Vanderschantz, N., \& Timpany, C. 2013. Who Says You Can't Judge a Book by its Cover? International Journal Of The Book, 10(4), 1-1. 


\section{SUPPLIMENTS}

We have used the visual representations of a series of real books within this paper. A cross section of one of the researchers personal physical libraries was selected for inclusion as a realistic set of books that may appear in a pDL. The covers and spines of these books were scanned and placed onto book representations using design software. These book representations were used within the paper prototypes for this study and we include these within our paper to assist the reader. These images of the book covers and spines have been used under Section 43 of the NZ Copyright Act (1994) and complete acknowledgement of copyright and appreciation, gratitude and thanks is given to the authors, publishers and copyright holders of these works.

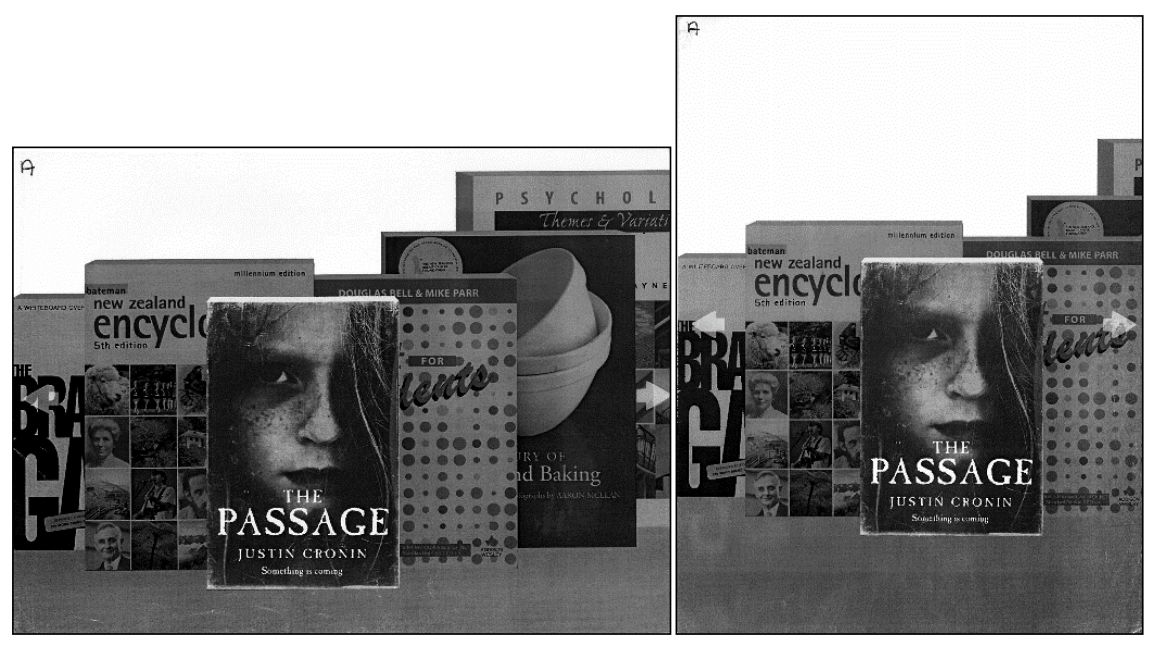

Figure A.1: Prototype A

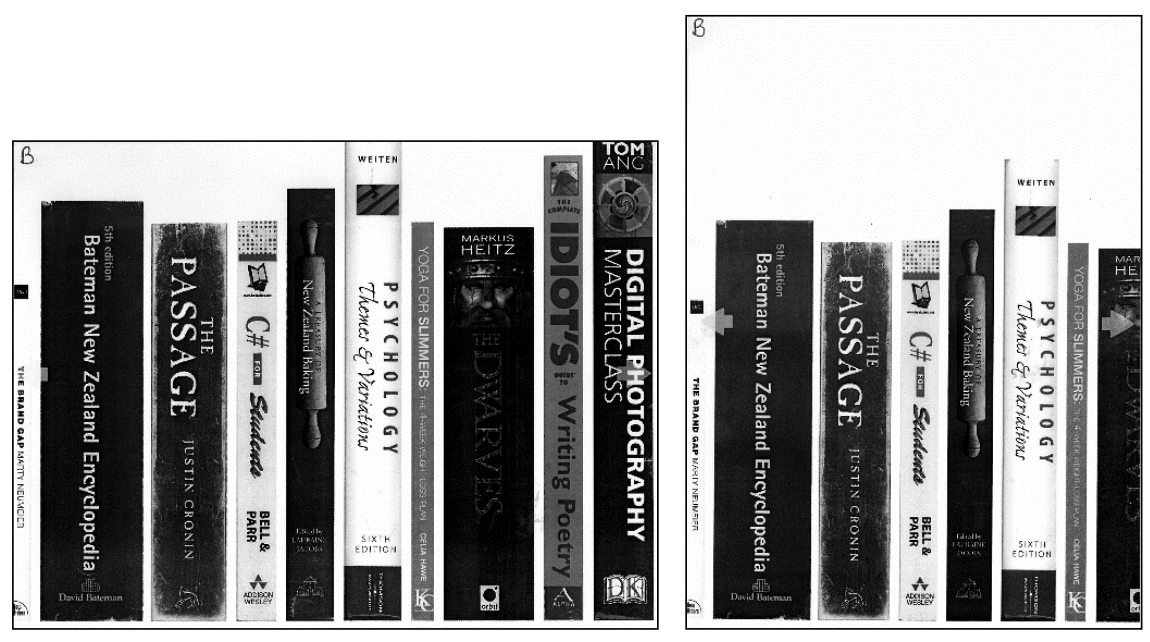

Figure A.2: Prototype B 


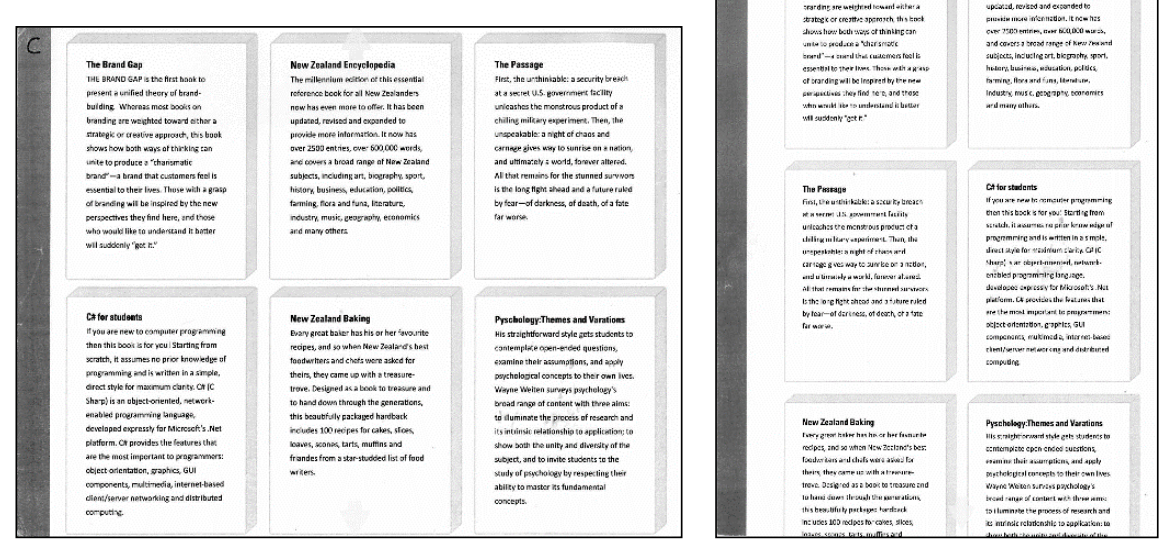

Figure A.3: Prototype C

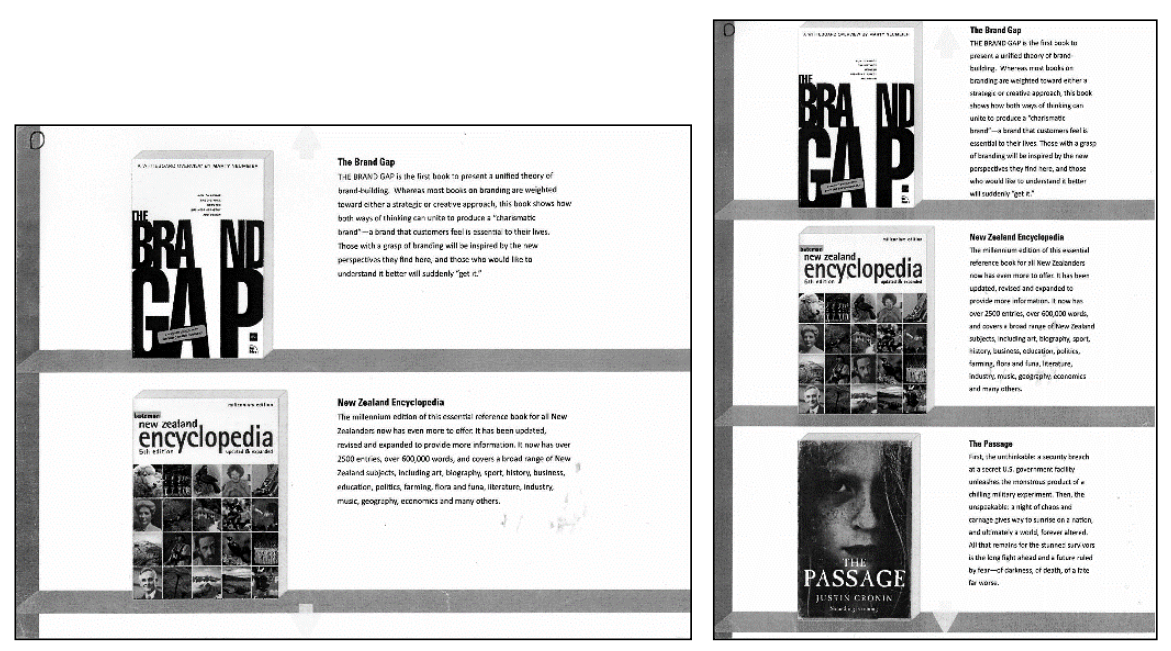

Figure A.4: Prototype D

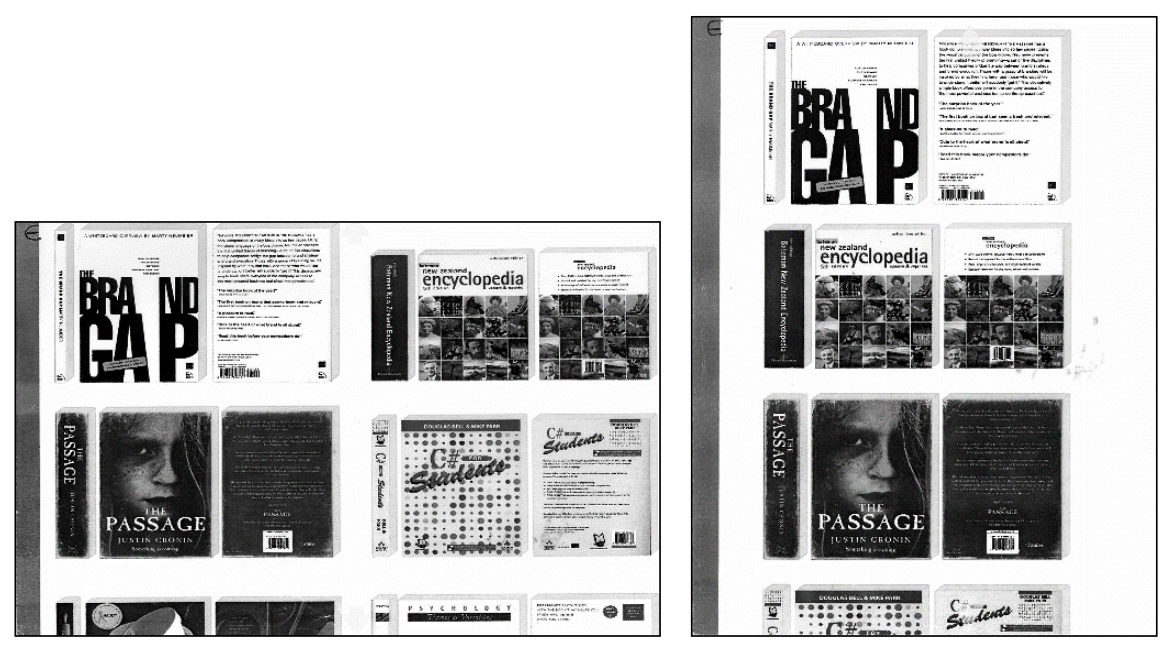

Figure A.5: Prototype E 